\title{
Avertissement au sujet des questions de mesure et de méthodologie associées au suivi et à l'évaluation du coronavirus dans l'ensemble des administrations
}

\author{
Robert Ladouceur ${ }^{1}$, Howard Shaffer ${ }^{2}$, Paige Shaffer ${ }^{3 *}$, Lucie Baillargeon ${ }^{4}$
}

\section{Résumé}

Alors qu'une pandémie dévastatrice affecte le monde entier, il est essentiel que les décideurs tiennent compte des problèmes de méthodologie et de mesure qui pourraient être associés aux indicateurs de santé publique de la maladie à coronavirus 2019 (COVID-19). Ce commentaire comprend quatre variables principales pour illustrer les questions de mesure et de méthodologie qui peuvent compliquer les comparaisons entre les administrations. L'administration désigne une variété de régions géographiques, comme un pays, un État, une province ou un territoire. Ces variables jouent un rôle essentiel dans la façon dont nous comprenons la trajectoire de la propagation de la maladie. Ces variables contribuent également à notre compréhension des stratégies de prévention et de leur efficacité connexe, reflétant l'impact de la COVID-19 sur les hôpitaux. Il est essentiel que les intervenants en santé publique et le public reconnaissent que ces quatre simples variables peuvent varier considérablement d'une administration à l'autre.

Citation proposée : Ladouceur R, Shaffer HJ, Shaffer PM, Baillargeon L. Avertissement au sujet des questions de mesure et de méthodologie associées au suivi et à l'évaluation du coronavirus dans l'ensemble des administrations. Relevé des maladies transmissibles au Canada 2021;47(7/8):326-9.

https://doi.org/10.14745/ccdr.v47i78a01f

Mots-clés : coronavirus, COVID-19, mesure, politique publique, santé publique, indicateurs

\section{Introduction}

Une pandémie dévastatrice affecte le monde entier. Le coronavirus s'est propagé partout dans le monde et la maladie associée au coronavirus 2019 (COVID-19) a touché diverses administrations. Personne n'a été épargné. Au moment où nous soumettons ce commentaire pour publication, il y a un total de 100746915 cas confirmés et 2170467 décès dans le monde (1). Les pathologistes et les experts médicaux ont travaillé à un rythme effréné pour mettre au point des vaccins et des traitements qui peuvent prévenir et contrer les conséquences de la COVID-19 et ses séquelles indésirables. En travaillant au sein d'équipes de collaboration avec des membres de partout dans le monde, la plupart des intervenants ne s'attendent pas à trouver une solution définitive dans les mois ou peut-être les années à venir, bien que des interventions prometteuses aient été cernées. Malgré ces efforts énergiques et enthousiastes, les secteurs gouvernementaux de la santé publique ont la responsabilité de prendre des décisions stratégiques urgentes en matière de santé publique afin de réduire et de prévenir les effets négatifs de la COVID-19. À titre d'exemple, des mesures de surveillance statistique ont été utilisées pour guider les efforts de dépistage et de test, ainsi que pour limiter les déplacements entre les administrations. Ces mesures ont été utilisées pour faire progresser les politiques publiques et protéger les travailleurs de la santé et les citoyens. La meilleure façon de protéger les citoyens en prévenant l'incidence de la COVID-19 est devenue I'un des problèmes de santé publique les plus urgents en ces temps difficiles, et cette situation demeurera pendant la phase de distribution de vaccins et par la suite, notamment en ce qui concerne la surveillance continue liée à la COVID-19.

Pendant leur processus décisionnel, les responsables des politiques publiques s'appuient sur des résultats scientifiques limités et en évolution pour orienter leur prise de décisions. Ces dirigeants recrutent des experts en santé publique, des épidémiologistes, des spécialistes des maladies infectieuses, des microbiologistes et d'autres experts pour aider à interpréter les constatations scientifiques et donner un aperçu d'un paysage de données probantes qui évolue rapidement. Cependant, étant donné que notre compréhension de la nature de ce virus et de ses conséquences est encore naissante dans la communauté 
scientifique, ces décisions sont difficiles et complexes. Par exemple, l'établissement de politiques sur la durée du confinement dans une collectivité est imprécis au mieux, en partie parce que les spécialistes des maladies infectieuses et les épidémiologistes ne comprennent pas pleinement la nature du virus, même si l'on connaît la période d'incubation.

Pour faciliter la prise de décisions, les décideurs se tournent souvent vers d'autres administrations ou d'autres pays pour évaluer leurs pratiques et leurs protocoles. Cette tendance est étroitement liée à diverses questions de méthodologie et de mesure. Des « expériences naturelles » qui comparent différentes politiques et approches du problème d'une région à l'autre sont certainement souhaitables. Pendant une pandémie, toutes les sources bien informées sont les bienvenues. Cependant, les analyses de ce qui se fait dans d'autres pays ou administrations laissent entendre que le processus de "comparaison » repose sur des données comparables. Bien qu'elles soient louables et nécessaires en ce moment critique, ces évaluations doivent être fondées sur une base commune de données probantes fiables et valides. Les preuves doivent être établies. Par exemple, il faut déterminer des variables pertinentes et représentatives pour faire des comparaisons entre les régions. De plus, il faut utiliser ces comparaisons à partir de variables qui sont décrites clairement et utilisées avec précision. Autrement, les comparaisons importantes sont discutables et, en fin de compte, comportent des lacunes. Enfin, il faut comparer des pommes avec des pommes, sinon nous risquons d'être induits en erreur.

L'objet de ce commentaire est de cerner certaines des variables clés qui fournissent aux décideurs les renseignements qu'ils pourraient utiliser pour protéger le public et suivre la propagation de la COVID-19. Le commentaire portera principalement sur ce corpus de données, en décrivant avec quelle facilité les observateurs peuvent obtenir une mauvaise interprétation. Le but est de discuter de ces variables fondamentales pour illustrer certains des problèmes associés aux preuves liées à la COVID-19. Toutefois, l'intention n'est pas de discuter longuement des questions méthodologiques soulevées dans le présent document. Il existe de nombreux textes de qualité pour guider ces méthodes d'enquête $(2,3)$. Le principal objectif de ce commentaire est de lancer un appel à faire preuve de prudence et de clarifier les nuances au sujet des comparaisons souvent faites entre les administrations et les pays.

Les analyses des impacts de la COVID-19 dans les médias et parmi les scientifiques tendent à donner une grande quantité de renseignements. Bien qu'il y ait de nombreuses variables qui méritent d'être prises en considération, une discussion sur toutes les mesures dépasse la portée de ce commentaire. La discussion se limitera donc aux quatre principales variables suivantes sélectionnées en partie parce que ces mesures de surveillance sont couramment déclarées par les médias dans une grande variété d'administrations :

1. Nombre de cas positifs de COVID-19

2. Cas hospitalisés

3. Cas dans les unités de soins intensifs des hôpitaux

4. Décès liés à la COVID-19

Ces variables sont des indicateurs principaux et précis des divers effets du coronavirus. La question centrale est de savoir « dans quelle mesure les intervenants peuvent-ils utiliser ces variables et les données probantes qu'elles génèrent pour établir des comparaisons solides, pertinentes et fiables entre les administrations "? I| est pertinent de répéter que la possibilité de comparer les données probantes entre les administrations associées à différentes politiques publiques offre une occasion importante de mener des recherches sur le caractère naturel. Au cours de l'examen qui suit, des questions clés seront soulevées au sujet de ces quatre variables qui tiendront compte de la façon dont les intervenants les utilisent lorsqu'ils comparent l'incidence de la COVID-19 entre différents pays ou d'autres administrations. De plus, des questions semblables seront soulevées au sujet des mesures préventives initiales que les enquêteurs mettent en œuvre pour atténuer les répercussions de la COVID-19.

\section{Nombre de cas positifs de COVID-19}

- Quelle est la définition d'un cas? Est-elle confirmée par un test normalisé ou par des symptômes cliniques associés à la COVID-19 avec ou sans contact à haut risque?

- La procédure de déclaration associée aux cas identifiés estelle la même d'une administration à l'autre?

- Combien de tests ont été effectués par milliers de personnes?

- La disponibilité et l'accessibilité des tests sont-elles semblables d'une administration à l'autre?

- Les médecins traitent-ils les cas de la même façon d'une administration à l'autre si les symptômes sont bénins?

- La validité des tests de dépistage (sensibilité et spécificité) est-elle identique d'une administration à l'autre?

- Le nombre de cas déclarés est-il fondé sur le même ratio (i.e. nombre de cas par milliers d'habitants)?

- Quelles sont les personnes testées? S'agit-il de bénévoles à risque, choisis au hasard?

- Les critères de sélection sont-ils les mêmes dans toutes les administrations?

\section{Cas hospitalisés}

- Les critères d'hospitalisation pour la COVID-19 sontils appliqués de façon identique dans toutes les administrations?

- L'accès aux hôpitaux est-il comparable d'une administration à l'autre?

- La disponibilité des hôpitaux est-elle identique d'une administration à l'autre?

- Le coût de l'hospitalisation est-il identique d'une administration à l'autre? 


\section{Cas dans les unités de soins intensifs}

- Les médecins qui travaillent dans les hôpitaux utilisent-ils les mêmes critères pour transférer un patient à l'unité de soins intensifs?

- La disponibilité des unités de soins intensifs dans les hôpitaux est-elle identique d'une administration à l'autre?

- Le coût d'une unité de soins intensifs reste-t-il le même d'une administration à l'autre?

\section{Décès causés par la COVID-19}

- Les procédures utilisées sont-elles les mêmes dans l'ensemble des administrations pour déterminer la cause d'un décès?

- Comment les autorités identifient-elles la COVID-19 comme cause de décès chez les patients souffrant d'autres problèmes médicaux (comorbidité)?

- Est-ce que tous les décès survenant dans différents lieux de service sont inclus (e.g. hôpitaux, établissements de soins de longue durée, résidence personnelle, etc.)?

- Le nombre de décès déclarés au cours d'une période donnée est-il complet, définitif et uniforme d'une administration à l'autre?

\section{Mise en œuvre de mesures préventives}

À l'heure actuelle, les décideurs utilisent trois principales mesures préventives pour comparer les influences de la COVID-19 entre les administrations, soit la distance physique, la quarantaine et l'utilisation de l'équipement de protection individuelle. Ces mesures ont donné lieu à des débats considérables et animés entre les administrations. Avant de faire des comparaisons entre les régions géographiques, il faut soulever les questions suivantes :

- Les procédures de confinement et de distanciation physique sont-elles opérationnalisées et appliquées de façon identique dans toutes les administrations?

- Les réseaux sociaux sont-ils conformes, confinés et distancés physiquement de la même façon d'une administration à l'autre?

- L'application de l'isolement et de la distance physique estelle identique d'une administration à l'autre?

- Les recommandations relatives à l'équipement de protection individuelle sont-elles semblables d'une administration à l'autre?

- La disponibilité de l'équipement de protection individuelle est-elle semblable d'une administration à l'autre?

Il est possible d'aborder les mêmes questions en ce qui concerne la procédure de mise en quarantaine. De plus, il est pertinent de soulever les questions suivantes:

- Sur quelle base les autorités gouvernementales ont-elles autorisé le confinement ou le déconfinement?

- Est-ce que cela a été permis de façon directe ou progressive?

- La surveillance a-t-elle été la même dans toutes les administrations?

\section{Discussion}

Dans ce commentaire, certaines des principales variables qui influent sur la production de données probantes liées à la COVID-19 dans l'ensemble des administrations sont décrites. Certaines variables et mesures connexes sont simples, mais les intervenants en appliquent d'autres de façon incohérente. Ces différences encouragent les comparaisons erronées et rendent difficile l'évaluation des politiques de santé publique. En comparant différents produits ou différentes procédures, l'accent porte sur le fait que la recherche méthodologique de base exige que la mesure de ces produits et de ces procédures soit identique, ou du moins très semblable.

Dans le cas de la crise du coronavirus, il est discutable et potentiellement dangereux d'affirmer qu'une administration réussit mieux ou moins bien qu'une autre lorsqu'elle est employée à des fins politiques. Les décideurs doivent garder à l'esprit que la comparaison entre les administrations quant à l'efficacité des méthodes de contrôle des répercussions de la COVID-19, sans savoir si les enquêteurs ont appliqué les mesures de façon identique ou semblable, pourrait ne pas être aussi informative que prévu ou, pire, trompeuse.

Par ailleurs, afin d'améliorer la mesure de la surveillance de la COVID-19, des experts nationaux et internationaux comme l'Organisation mondiale de la Santé pourraient proposer des mesures normalisées pour permettre des comparaisons entre les pays. Enfin, nous encourageons les intervenants en santé publique et le public à évaluer plus soigneusement ces données et leurs nuances lorsqu'ils interprètent les répercussions de la COVID-19 et en font rapport.

\section{Déclaration des auteurs}

Tous les auteurs ont approuvé la version finale envoyée pour publication et sont responsables de tous les aspects du travail.

Le contenu de l'article et les points de vue qui y sont exprimés n'engagent que les auteurs et ne correspondent pas nécessairement à ceux du gouvernement du Canada.

\section{Intérêts concurrents}

Les auteurs n'ont pas d'intérêts concurrents.

\section{Financement}

Aucun financement n'était associé à la préparation de ce manuscrit. 


\section{Références}

1. Johns Hopkins University School of Medicine. Corona Virus Resource Center. https://coronavirus.jhu.edu/map.html
2. Gordis L. Epidemiology. $5^{\text {th }}$ ed. Philadelphia, PA: Elsevier/ Saunders; 2014.

3. Shi L. Health Services Research Methods. $3^{\text {rd }}$ ed. Boston, MA: Cengage Learning; 2019.

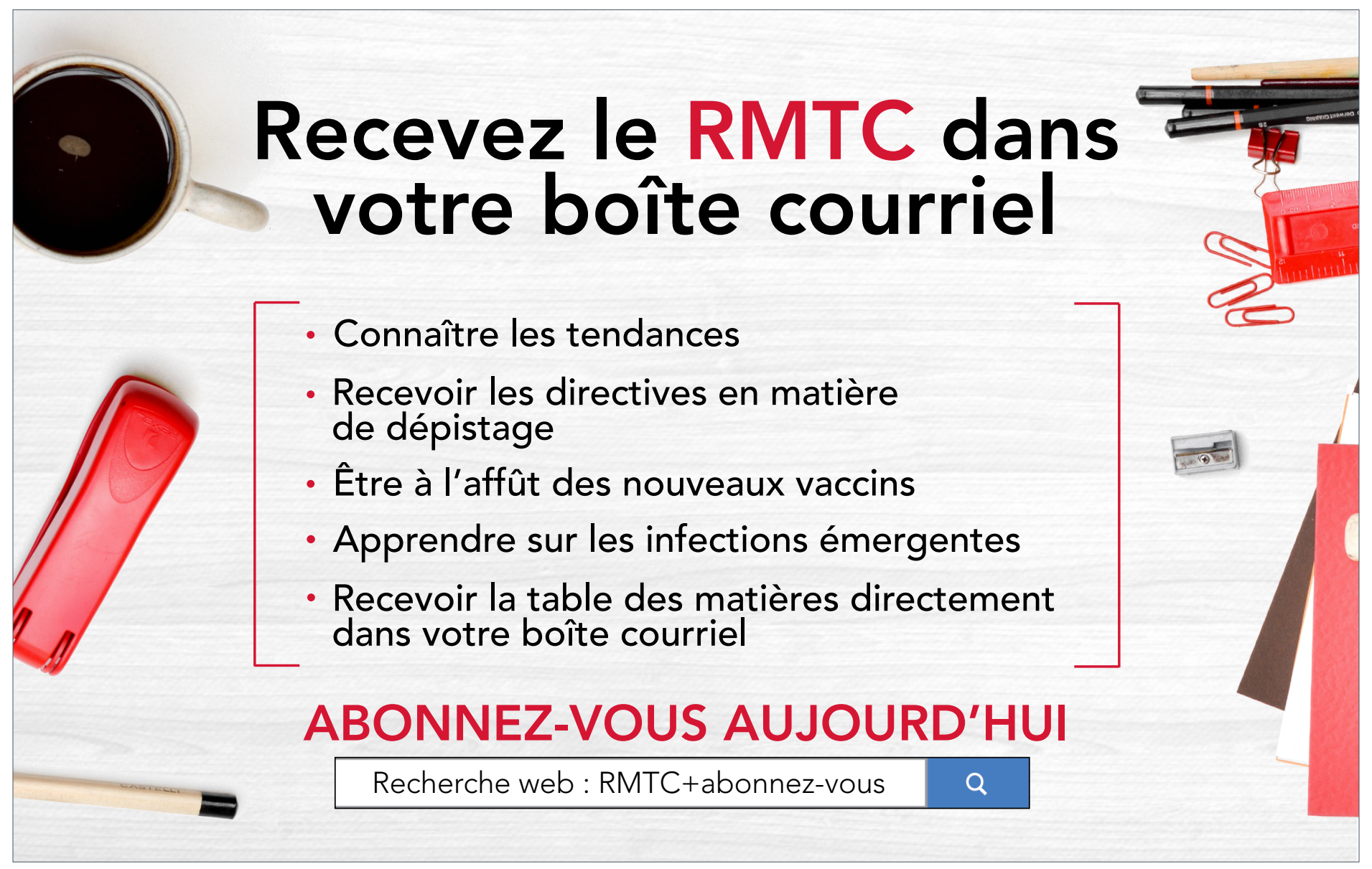

